\title{
Particle Patterning Based on Positive Dielectrophoresis Using a Scanning Microelectrode
}

\author{
Tomoyuki Yasukawa, ${ }^{1 *}$ Takuma Gotoh, ${ }^{1}$ Takashi Yasuda, ${ }^{2}$ \\ Masato Suzuki, ${ }^{1}$ and Fumio Mizutani ${ }^{1}$ \\ ${ }^{1}$ Graduate School of Material Science, University of Hyogo, \\ 3-2-1 Kouto, Kamigori, Ako, Hyogo 678-1297, Japan \\ ${ }^{2}$ Graduate School of Life Science and Systems Engineering, \\ Kyushu Institute of Technology, 2-4 Hibikino, Wakamatsu-ku, \\ Kitakyushu-shi, Fukuoka 808-0196, Japan
}

(Received June 29, 2018; accepted August 27, 2018)

Keywords: positive dielectrophoresis, particle manipulation, microelectrode

Positioning and patterning of polystyrene particles on a silicon nitride ( $\mathrm{SiN}$ ) membrane with a microhole array has been demonstrated by positive dielectrophoresis (p-DEP). A chamber with an SiN membrane with the well-aligned microholes as the bottom substrate was positioned on an indium-tin-oxide (ITO) electrode with a $1 \mathrm{~mm}$ space between the bottom substrate and the ITO electrode. The chamber and the space were filled with water and a suspension of particles $(10 \mu \mathrm{m}$ diameter) in water, respectively. An AC electric signal was then applied to a microelectrode positioned at $10 \mu \mathrm{m}$ above the SiN membrane, while the ITO electrode was connected to the ground. Particles present in the space between the SiN membrane and ITO electrode gradually moved toward the lower surface of the SiN membrane directly under the microelectrode owing to the strong electric field generated on and in localized microholes and accumulated at this position to form aggregates. For particles of $3 \mu \mathrm{m}$ diameter, one particle was deposited in each hole ( $2 \mu \mathrm{m}$ diameter) in the region directly under the microelectrode. The horizontal movement of the microelectrode gave rise to the formation of a line pattern of particles along the trail of the microelectrode because of the shift of the region with the strong electric field. These demonstrations could be applicable to arranging the particles at desired positions and in desired holes, and form particle patterns with highly flexible designs.

\section{Introduction}

Particle patterning by rapid and simple manipulation is of interest for developing a novel sensing platform. ${ }^{(1)}$ Dielectrophoresis (DEP) is an attractive scheme to form a pattern of particles on a substrate. ${ }^{(2,3)}$ DEP manipulation is fully controllable by varying the frequency and intensity of the applied electric field, and the electrical conductivity and permittivity of the suspending medium, because DEP forces originate from the difference in polarizability between a particle and its suspending medium. ${ }^{(4-6)}$ An attractive force to direct particles

*Corresponding author: e-mail: yasu@sci.u-hyogo.ac.jp

https://doi.org/10.18494/SAM.2019.2038 
toward a strong electric field region is called positive DEP (p-DEP), while a repulsive force from a strong electric field is called negative DEP (n-DEP). The force acts on almost all particles including electrically charged and uncharged particles and biological cells. Therefore, the manipulation based on DEP has been widely employed in the operation of Lab-on-a-Chip devices for various applications, such as the concentration and separation of bioparticles, and an accommodation of particles at the desired position. ${ }^{(7,8)}$ We have recently reported line patterning with microparticles and living cells. ${ }^{(9-11)}$ Microelectrode arrays embedded within microchannels were employed to generate a spatial gradient of an electric field. When an AC voltage was applied to the microelectrodes, particles and cells were rapidly manipulated to form line patterns reflecting the design of the template electrode by n-DEP. Patterned obstacles were also used to form a nonuniform electric field within the microchannels. ${ }^{(12-16)}$

Recently, micro- and nanohole arrays have been easily fabricated by micro- and nanofabrication techniques. ${ }^{(17)}$ The nanohole arrays are used fundamentally as plasmonic biosensors ${ }^{(18-21)}$ and nanochannels for selective injection of chemical stimuli and drug candidates. ${ }^{(22)}$ We previously fabricated a cell culture chamber consisting of a silicon nitride (SiN) membrane with a nanohole array in order to control the differentiation of cells cultured on the substrate selectively by releasing nerve growth factors in a localized region in the chamber. ${ }^{(23)}$ Microhole arrays were also applied to isolate specific cells rapidly and simply according to differences in size and deformability of cells. ${ }^{(24-26)}$ Individual cells were trapped on microcavities by applying a negative pressure. We have fabricated a microwell array electrode to introduce individual cells to microwells by p-DEP. ${ }^{(27)}$ The device with microwell arrays was applied to form pairs of different types of cells. ${ }^{(28)}$ More recently, gold nanohole arrays were combined with particle manipulation in order to enhance the performance of surface plasmon resonance sensors in detecting biomolecules. ${ }^{(29)}$ It is, however, difficult to draw the pattern with particles in the localized and selected holes on the substrate by these techniques. Electric fields generated cannot be selectively regulated in individual microwells because of the fabrication of microwells on a bare conductive substrate. Therefore, we cannot individually manipulate particles in the respective microwells.

In this work, we described the manipulation of particles at the localized area on the substrate with microhole arrays by applying a needle-type microelectrode. Previously, patterns with particles on and in the porous membranes have been formed by p-DEP using devices consisting of a disk array electrode and a bare electrode. ${ }^{(30,31)}$ When we applied AC voltage between the disk array electrode and the bare electrode, strong electric fields were formed on and in holes in the porous membrane, resulting in the particles moving on and in holes to form an island organization with particles. However, we cannot arrange particles at any positions on a porous membrane. The techniques for positioning particles and living cells at any positions on the membrane and collecting targeted particles and cells on the membrane is useful to develop novel cocultivation systems and sensing platforms. In this work, an AC electric signal was applied to the microelectrode at $10 \mu \mathrm{m}$ above a SiN membrane with highly ordered microholes to accumulate particles on the lower surface of the SiN membrane directly under the microelectrode by the attractive force of p-DEP. The particles of $3 \mu \mathrm{m}$ diameter were precisely deposited in the holes ( $2 \mu \mathrm{m}$ diameter) in the region directly under the microelectrode. 
Moreover, the horizontal movement of the microelectrode gave rise to the formation of the line pattern with particles along the trail of the microelectrode because of the shift of the region with the strong electric field. We demonstrated that the development of these techniques would be useful for arranging the particles at the desired position and in the desired holes, and form particle patterns with highly flexible designs.

\section{Materials and Methods}

\subsection{Fabrication of DEP device for patterning particles}

The DEP device consists of a chamber with a microhole array, an indium-tin-oxide (ITO) electrode, and a needle-type Pt microdisk electrode. Figure 1(a) shows cross-sectional and top illustrations of the chamber. The microhole array was fabricated on a SiN diaphragm $(1.0 \mu \mathrm{m}$ thick and $9.0 \mathrm{~mm}$ diameter) supported by a Si ring frame ( $300 \mu \mathrm{m}$ thick, $9.0 \mathrm{~mm}$ inner diameter, and $12 \mathrm{~mm}$ outer diameter) by photolithography and dry etching with $\mathrm{CF}_{4}$ plasma. Figure 1(b) shows the optical microscopy image of the microhole array. Each microhole was $2.0 \mu \mathrm{m}$ in diameter and at a distance of $5.0 \mathrm{~mm}$ from adjacent holes. Cylindrical resin (approximately $9 \mathrm{~mm}$ height, $9.0 \mathrm{~mm}$ inner diameter, and $12 \mathrm{~mm}$ outer diameter) was attached to the Si support for use as a chamber. The chamber with three resinous piers $(1 \mathrm{~mm}$ height $)$ was then mounted on the ITO electrode with a $1 \mathrm{~mm}$ space between the bottom of the chamber and the ITO surface as shown in Fig. 1(c).

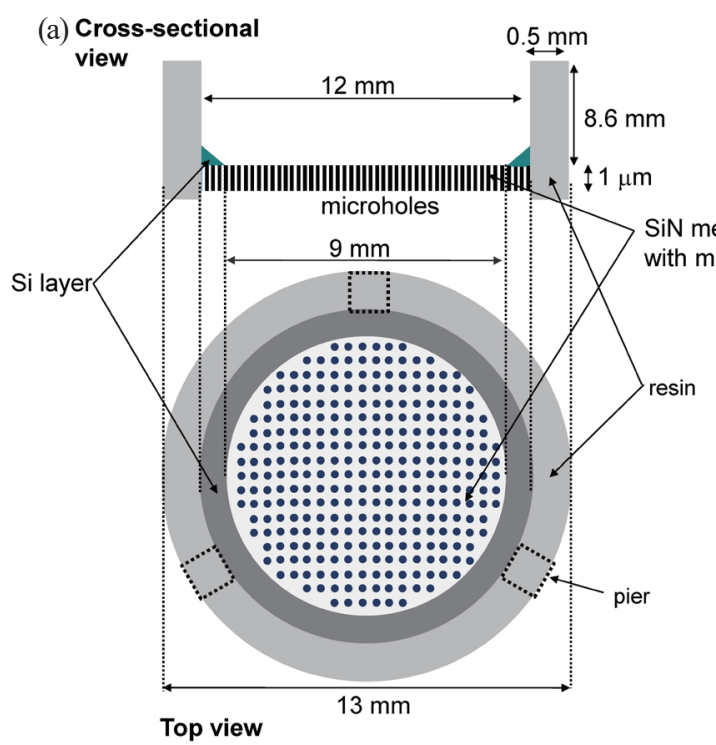

(b)
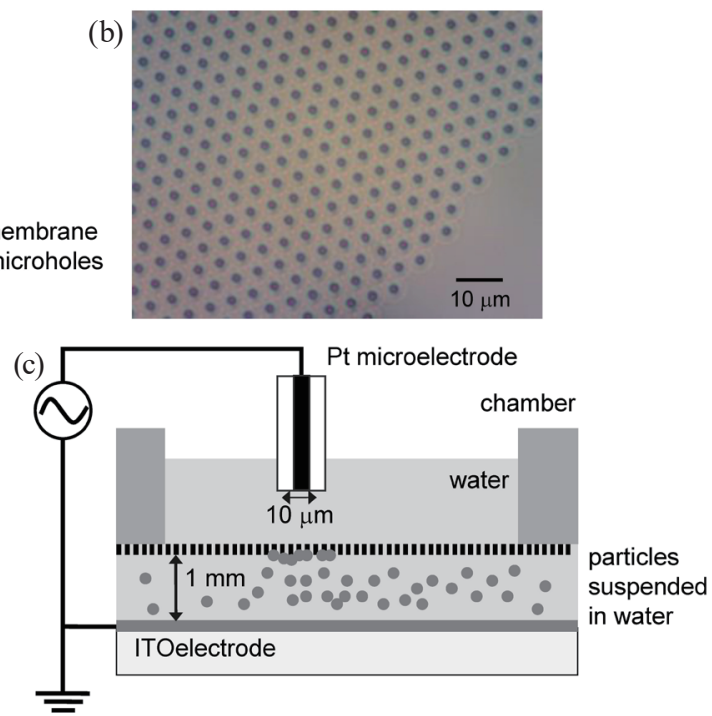

Fig. 1. (Color online) (a) Cross-sectional and top illustrations of the chamber. (b) Optical microscopy image of microhole array. (c) Structure of the DEP device consisting of a chamber with a microhole array, an ITO electrode and a needle-type Pt microdisk electrode. 


\subsection{Line patterning with particles using p-DEP}

Water and a suspension of polystyrene particles $\left(10\right.$ or $3 \mu \mathrm{m}$ diameter, $2.7 \times 10^{7}$ particles $\mathrm{mL}^{-1}$ ) were introduced into the chamber and the space between the SiN membrane with microholes and the ITO surface, respectively. To prepare the microdisk electrode, a Pt wire (10 $\mu \mathrm{m}$ diameter) was inserted into a glass capillary, and the tip region was thermally fused and polished. A Pt microelectrode was adopted on a XYZ manipulator (Suruga Seiki, Tokyo, Japan) and positioned $10 \mu \mathrm{m}$ above the SiN membrane with microholes. Particle manipulation via p-DEP was performed under an optical microscope (Eclipse, Ti-S, Nikon Corp., Tokyo, Japan) equipped with a CCD camera (Moticam 2000, Shimadzu Corp., Kyoto, Japan). An AC voltage [typically 400 or $500 \mathrm{~V}$ peak to peak $\left(\mathrm{V}_{\mathrm{pp}}\right)$ ] in the p-DEP frequency region $(1.0 \mathrm{kHz})$ was applied to the microelectrode in order to form the nonuniform electric field in the device using a function generator (1956, NF Corp., Yokohama, Japan), while the ITO electrode was connected to the ground. The microelectrode was scanned at $10 \mu \mathrm{m} \mathrm{s}^{-1}$ in the horizontal direction at a constant height from the SiN membrane with microholes to form the line pattern of particles on the lower side of the SiN membrane.

\section{Results and Discussion}

\subsection{Accumulation of particles by p-DEP}

p-DEP-based accumulation of microparticles was studied using the DEP device presented in Fig. 1. A particle suspension was injected into the device in which the microelectrode was used to manipulate the suspended particles to the lower surface of the SiN membrane through the attractive force arising from p-DEP. Figure 2 depicts sequential images of the particles accumulated using p-DEP. The application of a $1.0 \mathrm{kHz}$ AC voltage $(500 \mathrm{Vpp})$ to the Pt microdisk forced the particles to move to the SiN membrane. The three-dimensional distribution of the electric field strength was calculated by the finite element method (FEM) solver using the macroscopic and microscopic models in the previous reports. ${ }^{(30,31)}$ The voltage was applied between the upper bare electrode and lower electrode with microdisks. The regions with high electric field were formed in the areas above the disks. Thus, the suspended particles move to the areas above the disks by p-DEP. In the calculation of electric field strength around the membrane with micropores above the disks based on a microscopic model, the electric fields formed in and around the pores are strong compared with those in the bulk solution. Therefore, in the present study, strong electric fields were formed in and around microholes under the microelectrode, resulting in the movement of particles dispersed in the space between the membrane and the ITO substrate toward the holes.

No particles were accumulated on the $\mathrm{SiN}$ membrane before $\mathrm{AC}$ voltage was applied [Fig. 2(a)], while the particles were accumulated on the SiN membrane in the region directly under the microelectrode through the attractive force of p-DEP after applying AC voltage to the microelectrode [Fig. 2(b)]. The first particle arrived at the SiN membrane 2 min after the application of voltage, and the number of accumulated particles then increased up to 6 


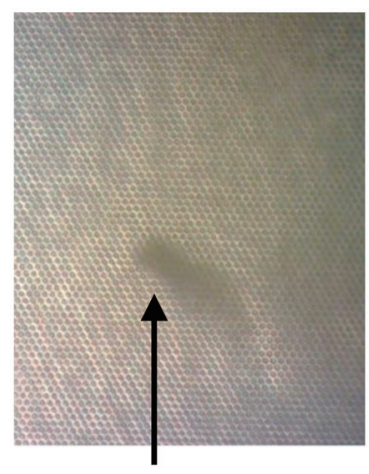

microelectrode

(a)

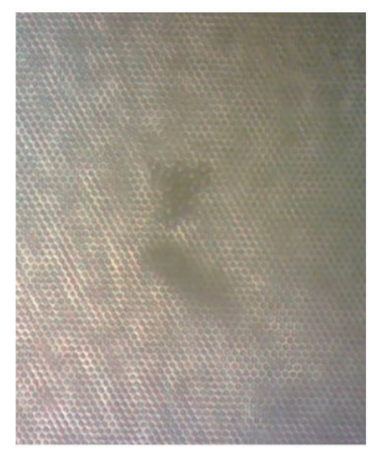

(b)

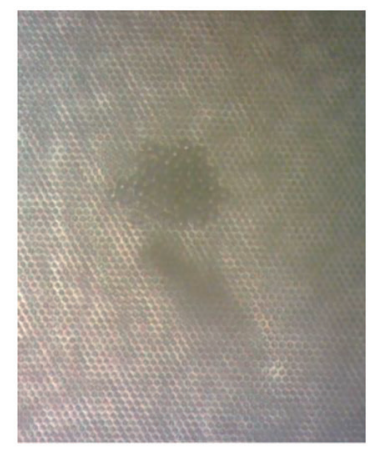

(c)

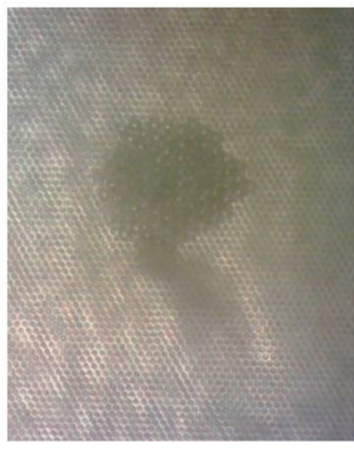

(d)

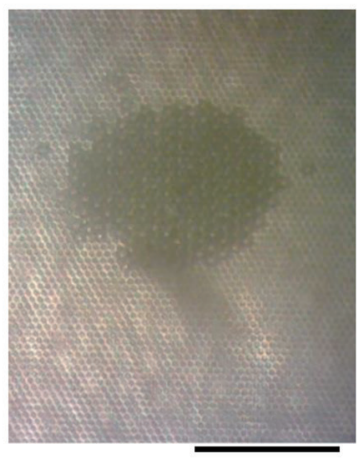

(e)

$100 \mu \mathrm{m}$

Fig. 2. (Color online) Sequential images of particles accumulating at the lower surface of the SiN membrane of the chamber owing to p-DEP (a) before and (b) 2.5, (c) 3, (d) 4, and (e) 6 min after the application of AC voltage.

min of applying AC voltage [Figs. 2(b)-2(e), Supporting Information Movie 1 (https://www. youtube.com/watch? $\mathrm{v}=\mathrm{pKFN} 26 \mathrm{mA6N0}$ ) reproduced at $\times 8$ speed]. Particles arriving at the $\mathrm{SiN}$ membrane were successively arranged at the periphery of the particle aggregation. The diameter of the particle aggregation increased at an average rate of $45 \mu \mathrm{m} \mathrm{min}^{-1}$ during 2 and 6 min after the application of voltage, and reached $180 \mu \mathrm{m}$ after $6 \mathrm{~min}$, but no increase in diameter was observed beyond $6 \mathrm{~min}$. This result may be due to the decrease in the electric field with increasing distance from the tip of the microelectrode. In addition, aggregated particles redispersed after switching off the $\mathrm{AC}$ voltage. Thus, the formation of particle aggregations was controlled by switching the applied voltage.

Figure 3 shows the microparticles on the ITO electrode before and 1 min after AC voltage was applied. Particles present on the surface of the ITO electrode directly under the microelectrode were in focus before voltage application [Fig. 3(a)]. However, particles became out of focus gradually after the AC voltage was applied [Fig. 3(b), Supporting Information Movie 2 (https://www.youtube.com/watch? $\mathrm{v}=\mathrm{D} 45 \mathrm{hfFc} 2 \mathrm{FxM}$ ) reproduced by $\times 8$ speed]. Particles on the ITO surface moved upward through the $1 \mathrm{~mm}$ space between the SiN membrane and the ITO electrode owing to the p-DEP. However, particles that located in areas other than 


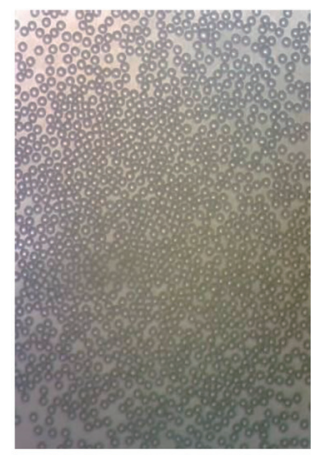

(a)

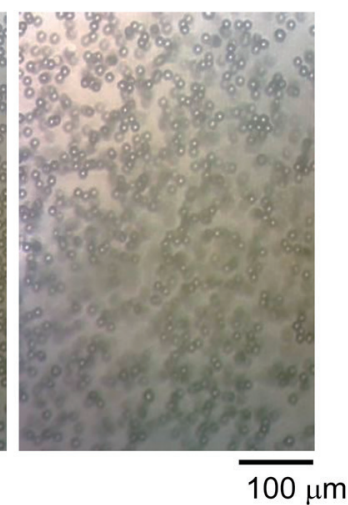

(b)

Fig. 3. (Color online) Microparticles on the ITO electrode (a) before and (b) 1 min after AC voltage was applied.

the area under the microelectrode stayed at the original position. Therefore, particles located in the region directly under the microelectrode were moved and aggregated, because a strong electric field was formed between the microelectrode and the bottom substrates in the region under the microelectrode.

\subsection{Formation of particle pattern by scanning microelectrode}

The particles were aligned in the regions along the trail of microelectrode movement. Figure 4 shows the line pattern of particles formed on the lower surface of the SiN membrane. A microelectrode with an applied AC voltage of $400 \mathrm{~V}_{\mathrm{pp}}$ was horizontally scanned $10 \mu \mathrm{m}$ above the SiN membrane at $10 \mu \mathrm{m} \mathrm{s}^{-1}$. The shadow of the microelectrode is seen on the left of the image in Fig. 4(a). When the microelectrode moved to the right, the particles arriving at the substrate were arranged to the right of the aggregation, resulting in the formation of the line pattern of particles [Figs. 4(b) and 4(c), Supporting Information Movie 3 (https://www.youtube. $\mathrm{com} /$ watch? $\mathrm{v}=\mathrm{PF} 75 \mathrm{pCc} 3 \mathrm{abw})]$. The region with the strong electric field was shifted along the movement of the microelectrode owing to the shift of microholes with the smallest distance between the microelectrode and the ITO electrode. The aligned pattern (four or five particles wide) was fabricated under the experimental conditions. It is noteworthy that the aligned particles remained on the SiN membrane after the microelectrode moved through. However, the particles aligned approximately $450 \mu \mathrm{m}$ from the position of the microelectrode started to move from those positions to the left, thereby decreasing the number of particles forming the line pattern [dotted square in Figs. 4(c) and 4(d)]. This phenomenon could be due to the decrease in the p-DEP force holding the particles upon the increase in the distance between the probe microelectrode and the aligned particles. In addition, the pattern of aligned particles almost disappeared 1 min after the AC voltage was turned off. We can control the duration of contact of particles with the membrane surface. Thus, there are possibilities to apply this redispersion of patterned particles to the determination of reaction rates of molecules immobilized on both particles and the membrane, and immunosensing by capturing patterned particles to 


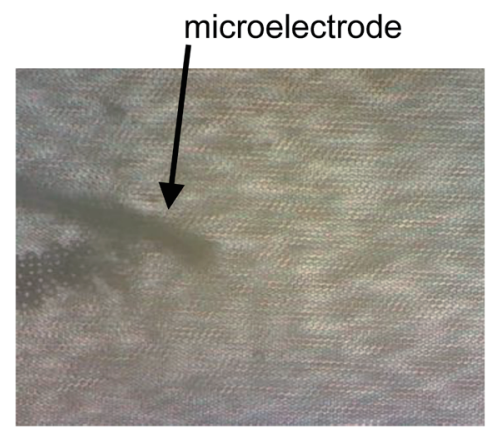

(a)

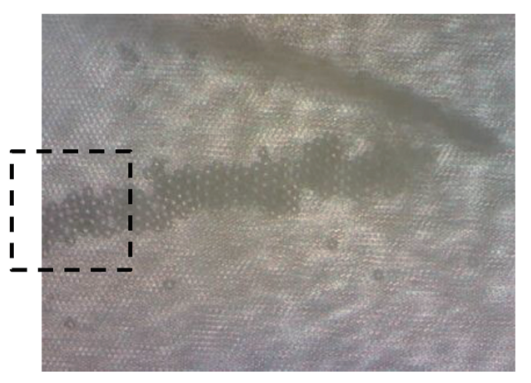

(c)

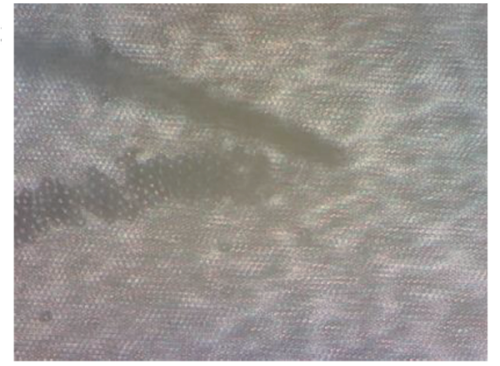

(b)

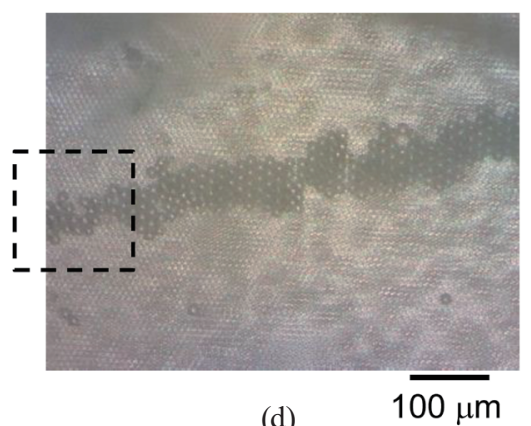

(d)

Fig. 4. (Color online) Line pattern of particles formed on the lower surface of the SiN membrane by scanning the microelectrode (400 Vpp) horizontally at $10 \mu \mathrm{m} \mathrm{s}^{-1}$. Patterns obtained after (a) 0, (b) 15, (c) 30, and (d) $45 \mathrm{~s}$.

form immunocomplexes between particles and the membrane modified with antibodies. The scanning microelectrode acts as the probe for particle patterning on demand. The combination of the chemical bonding reactions and biological recognition reactions could be useful in obtaining particles aligned on the SiN membrane at will.

\subsection{Particle trapping in microwells by p-DEP}

Dielectrophoretic trapping of particles $(3 \mu \mathrm{m}$ diameter) in the microwell array was studied using the DEP device. A suspension of microparticles was introduced into the device, and an $\mathrm{AC}$ voltage was subsequently applied to guide the particles to the microwells by p-DEP. Microscopy images after the application of the voltage are shown in Fig. 5 (Supporting Information Movie 4 (https://www.youtube.com/watch?v=ULWUEThjI-w) reproduced at $\times 16$ speed). A few particles were positioned into the holes before the application of the voltage [Fig. 5(a)]. On applying AC voltage $(400 \mathrm{Vpp})$, dispersed particles began to move toward the SiN membrane of the chamber and arrived 3 min after the application of voltage [Fig. 5(b)]. White circles observed in the image of the SiN membrane were gradually replaced by black circles [Fig. 5(b)-5(g)]. This indicates that the particles were trapped within the microholes by p-DEP. Figure 5(h) shows the occupation efficiency of the particles in the microwells, defined as the ratio of the number of microholes with particles to the total number of microholes as a function of time after the application of voltage. The reason for the initial occupation of the particles in 


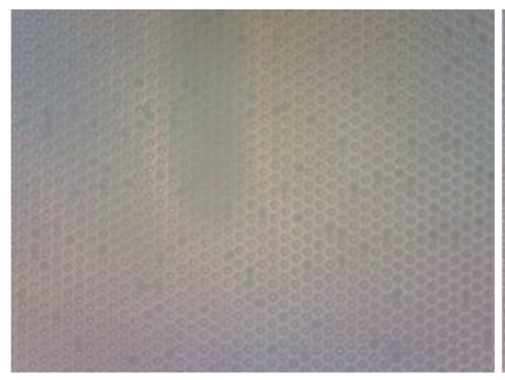

(a)

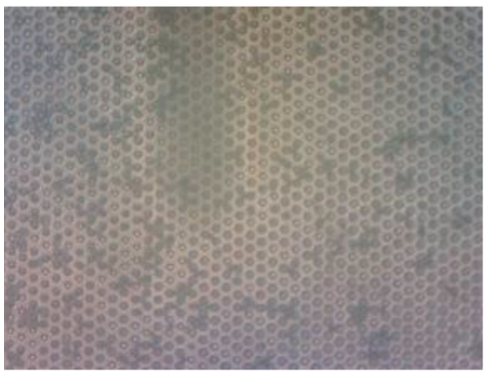

(d)

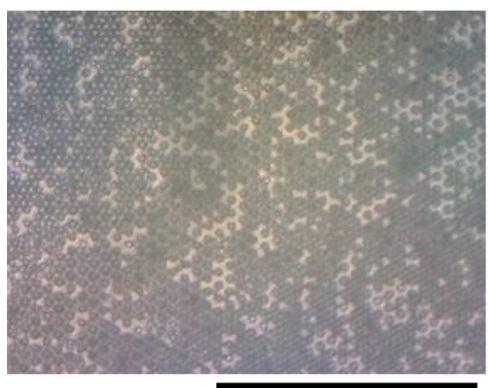

$100 \mu \mathrm{m}$

(g)

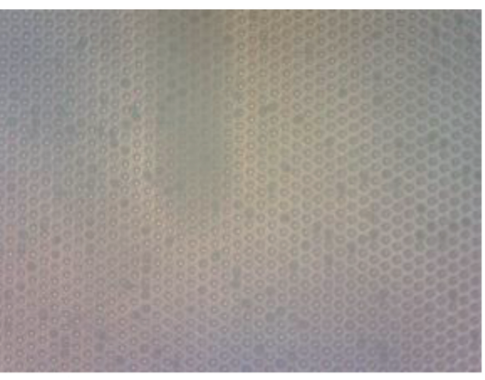

(b)

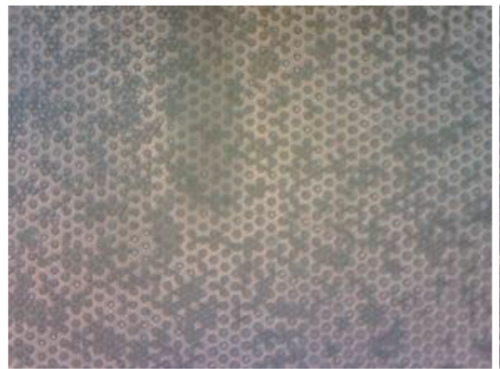

(e)

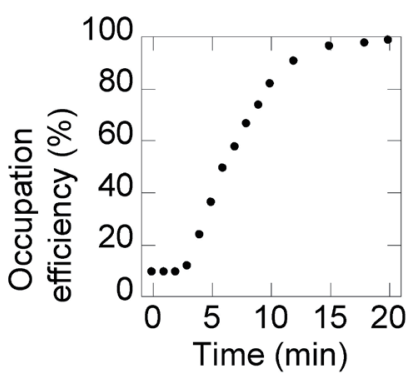

(h)

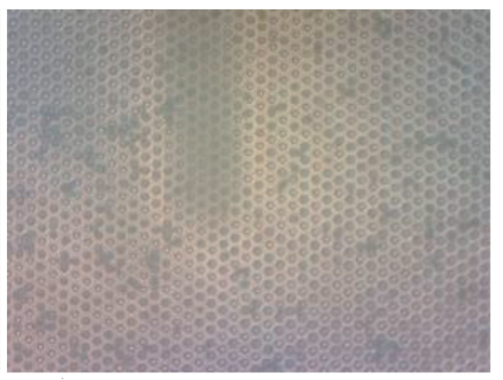

(c)

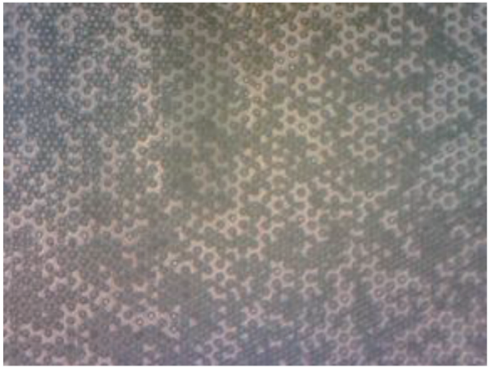

(f)

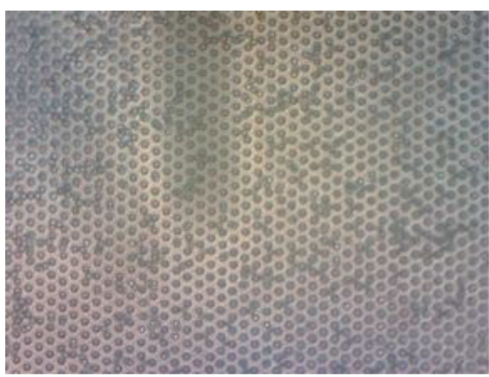

(i)

Fig. 5. (Color online) Series of images of trapping of the particles within the microholes obtained (a) before and (b) 3 , (c) 6, (d) 9, (e) 12, (f) 15, and (g) 18 min after application of voltage. (h) Occupation efficiency of the particles in the microwells as a function of time after application of voltage. (i) Particles trapped within microholes 1 min after the voltage was switched off.

the microholes is the flow of particle suspension from the lower side of the SiN membrane to the upper chamber upon injecting the particle suspension. The occupation efficiency increased 3 min after the application of voltage and was over $95 \%$ after $15 \mathrm{~min}$. The number of particles trapped in microholes did not increase without the p-DEP attractive force in this experimental period. Unfortunately, many particles were deposited in microholes on the SiN membrane other than the desired microholes because of interference by the trapped particles. However, the particles trapped within the microholes remained even after switching off the applied voltage, while most of the particles deposited other than those in the microholes were removed after 1 min [Fig. 5(i)]. This may be due to the larger contact region of the trapped particles for 
physical adsorption than that of particles deposited at undesired positions. These procedures for patterning and trapping particles by p-DEP will be applicable to the integration of various sensing devices.

\section{Conclusions}

A novel process was developed for the manipulation of particles at a localized area on the membrane with microhole ( $2 \mu \mathrm{m}$ diameter) arrays based on p-DEP by using a needle-type microelectrode. When $\mathrm{AC}$ voltage was applied to the microelectrode, strong electric fields were formed in the microholes of the SiN membrane in the region directly under the microelectrode, resulting in the particles moving toward the lower surface of the region through the attractive force of $\mathrm{p}$-DEP to form aggregates. Particles with $3 \mu \mathrm{m}$ diameter, which accumulated at the region under the microelectrode, also accumulated at the region to be occupied within the individual microholes by p-DEP. Although particles were deposited in microholes on the bottom substrate other than the desired microholes, the deposited particles were removed 1 min after the $\mathrm{AC}$ voltage was turned off to obtain particle patterns reflecting highly ordered microholes. Moreover, the scanning of the microelectrode applied with AC voltage resulted in the formation of a line pattern of particles along the trail of microelectrode movement owing to the shift of microholes by the strong electric field. Thus, the present methods are useful for arranging the particles at the desired position and in the desired holes, and fabricating patterns of particles on demand without using templates with chemical properties, and electrode and obstacle arrays. The process could be applied to the manipulation of living cells and bacteria. By applying the process to living cells and bacteria, we could easily fabricate island arrays and line patterns of different types of cells to develop a platform for cell-based assays and obtain the method of adding the patterns of cells to the patterns of the different types of cells formed on the membrane. This assembly procedure could have a wide variety of applications in the fabrication of patterned particles.

\section{Acknowledgments}

This work was partly supported by JSPS KAKENHI Grant Numbers 17H03468 and $16 \mathrm{H} 04169$.

\section{References}

1 N. Vogel, M. Retsch, C.-A. Fustin, A. del Campo, and U. Jonas: Chem. Rev. 115 (2015) 6265.

2 K. Khoshmanesh, S. Nahavandi, S. Baratchi, A. Mitchell, and K. Kalantar-zadeh: Biosens. Bioelectron. 26 (2011) 1800.

3 P. Y. Chiou, A. T. Ohta, and M. C. Wu: Nature 436 (2005) 370.

4 H. A. Pohl: Dielectrophoresis (Cambridge University Press, Cambridge, U.K., 1978).

5 T. B. Jones: Electromechanics of Particles (Cambridge University Press, New York, 1995).

6 H. Morgan and N. G. Green: AC Electrokinetics: Colloids and Nanoparticles (Research Studies Press Ltd., Baldock, Hertfordshire, England, 2003).

7 P. R. C. Gascoyne and J. Vykoukal: Electrophoresis 23 (2002) 1973.

8 M. P. Hughes: Electrophoresis 23 (2002) 2569. 
9 M. Suzuki, T. Yasukawa, Y. Mase, D. Oyamatsu, H. Shiku, and T. Matsue: Langmuir 20 (2004) 11005.

10 M. Suzuki, T. Yasukawa, H. Shiku, and T. Matsue: Langmuir 23 (2007) 4088.

11 M. Suzuki, T. Yasukawa, H. Shiku, and T. Matsue: Biosens. Bioelectron. 24 (2008) 1043.

12 B. H. Lapizco-Encinas, B. A. Simmons, E. B. Cummings, and Y. Fintschenko: Anal. Chem. 76 (2004) 1571.

13 B. H. Lapizco-Encinas, R. V. Davalos, B. A. Simmons, E. B. Cummings, and Y. Fintschenko: J. Microbiol. Methods 62 (2005) 317.

14 B. H. Lapizco-Encinas, B. A. Simmons, E. B. Cummings, and Y. Fintschenko: Electrophoresis 25 (2004) 1695.

15 E. B. Cummings and A. K. Singh: Anal. Chem. 75 (2003) 4724.

16 B. G. Hawkins, A. E. Smith, Y. A. Syed, and B. J. Kirby: Anal. Chem. 79 (2007) 7291.

17 V. Escobedo: Lab Chip 13 (2013) 2445.

18 A. G. Brolo, R. Gordon, B. Leathem, and K. L. Kavanagh: Langmuir 20 (2004) 4813.

19 A. D. Leebeeck, L. K. S. Kumar, V. de Lange, D. Sinton, R. Gordon, and A. G. Brolo: Anal. Chem. 79 (2007) 4094.

20 A. Lesuffleur, H. Im, N. C. Lindquist, K. S. Lim, and S. H. Oh: Opt. Express 16 (2008) 219.

21 H. Im, A. Lesuffleur, N. C. Lindquist, and S. H. Oh: Anal. Chem. 81 (2009) 2854.

22 B. L. Pioufle, P. Surbled, H. Nagai, Y. Murakami, K. S. Chun, E. Tamiya, and H. Fujita: Mater. Sci. Eng., C 12 (2000) 77.

23 Y. Nakashima and T. Yasuda, Sens. Actuators, A 139 (2007) 252.

24 T. Matsunaga, M. Hosokawa, A. Arakaki, T. Taguchi, T. Mori, T. Tanaka, and H. Takeyama: Anal. Chem. 80 (2008) 5139.

25 M. Hosokawa, A. Arakaki, M. Takahashi, T. Mori, H. Takeyama, and T. Matsunaga: Anal. Chem. 81 (2009) 5308.

26 M. Hosokawa, T. Hayata, Y. Fukuda, A. Arakaki, T. Yoshino, T. Tanaka, and T. Matsunaga: Anal. Chem. 82 (2010) 6629.

27 Y. Yoshimura, C. Fujii, M. Tomita, F. Mizutani, and T. Yasukawa: Chem. Lett. 43 (2014) 980.

28 Y. Yoshimura, M. Tomita, F. Mizutani, and T. Yasukawa: Anal. Chem. 86 (2014) 6818.

29 A. Barik: Nano Lett. 14 (2014) 2006.

30 H. J. Lee, T. Yasukawa, M. Suzuki, Y. Taki, A. Tanaka, M. Kameyama, H. Shiku, and T. Matsue: Sens. Actuators, B 131 (2008) 424.

31 H. J. Lee, T. Yasukawa, M. Suzuki, S. H. Lee, T. Yao, Y. Taki, A. Tanaka, M. Kameyama, H. Shiku, and T. Matsue: Sens. Actuators, B 136 (2009) 320. 